\title{
PERTURBATIONS DE QUELQUES PROPRIÉTÉS VIBRATIONNELLES DES SURFACES CRISTALLINES PAR LES MARCHES
}

\author{
P. MASRI $(*)$, G. ALLAN $(* *)$ et L. DOBRZYNSKI $(* *)$
}

$\left({ }^{*}\right)$ Service de Physique Atomique. Section d'Etudes des Interactions Gaz-Solides

C. E. N./Saclay, B. P. no 2, 91-Gif-sur-Yvette, France

(**) Equipe de Recherches du C. N. R. S., Physique des Solides, I. S. E. N., 3, rue François-Baës, 59-Lille, France

\author{
(Reçu le 7 juillet 1971)
}

\begin{abstract}
Résumé. - On étudie les perturbations produites par des marches [100] sur quelques propriétés vibrationnelles d'un cristal cubique simple de Montroll-Potts avec surface plate (001): perturbations de la densité d'états, variations de l'entropie $S$, de la chaleur spécifique à volume constant $C_{\mathrm{v}}$, de l'énergie libre $F$ et du déplacement carré moyen des atomes. Les calculs sont conduits au moyen de deux méthodes : la première utilise les fonctions de Green, associées à la méthode des déphasages ; la seconde, très simple et analytique, utilise la méthode des moments. Les résultats obtenus donnent l'ordre de grandeur de l'effet des marches sur quelques propriétés vibrationnelles des surfaces cristallines : par atome de coin de marche, on trouve une augmentation de l'entropie $S \simeq 0,39 k_{\mathrm{B}}$ à $T / T_{\mathrm{D}}=1,5$, de la chaleur spécifique $C_{\mathrm{V}} \simeq 0,23 k_{\mathrm{B}}$ à $T / T_{\mathrm{D}}=0,16$, diminution de l'énergie libre $F \simeq 0,6 k_{\mathrm{B}} T_{\mathrm{D}}$ à $T / T_{\mathrm{D}}=1,5$. Le rapport de la moyenne quadratique du déplacement d'un atome au coin d'une marche à celui d'un atome sur une surface (001) plate, perpendiculairement à cette surface, est $\simeq 1,3$, pour les températures comparables à la température de Debye $T_{\mathrm{D}}$.
\end{abstract}

\begin{abstract}
The perturbations to some vibrational properties - i. e. the density of states, the entropy $S$, the specific heat $C_{\mathrm{v}}$, the free energy $\mathrm{F}$ and the mean square displacement of atoms - of a simple cubic Montroll-Potts crystal with a (001) flat surface due to [100] steps have been studied. The calculations are made by two methods. The first method uses the Green functions associated with the phase shift technique. The second method which is very simple and analytic uses the method of moments. The results obtained give the order of magnitude of the step effect in some vibrational properties of crystal surfaces : for one atom at a corner step, we have an increase of entropy $S \simeq 0.39 k_{\mathrm{B}}$ for $T / T_{\mathrm{D}}=1.5$, an increase of specific heat $C_{\mathrm{v}} \simeq 0.23 k_{\mathrm{B}}$ for $T / T_{\mathrm{D}}=0.16$, a decrease of free energy $F \simeq 0.6 k_{\mathrm{B}} T_{\mathrm{D}}$ for $T / T_{\mathrm{D}}=1.5$. The ratio of the mean square displacement of a corner step atom to the mean square displacement of an atom on a (001) flat surface, in the direction normal to this surface, is $\simeq 1.3$, for temperatures which are comparable to the Debye temperature $T_{\mathrm{D}}$.
\end{abstract}

1. Introduction. - La surface d'un solide abaisse les fréquences des vibrations en volume, causant l'apparition d'ondes de surface et des variations de la densité des états de volume. En conséquence, elle doit contribuer, de façon spécifique, aux propriétés vibrationnelles du solide. Dans l'approximation de Debye, la distribution, en fréquence $v$, des phonons de volume est proportionnelle à $v^{2}$ avec une fréquence de coupure $v_{\mathrm{D}}$ dite fréquence de Debye. Dans cette approximation, aux basses températures, la contribution des phonons à la chaleur spécifique à volume constant $C_{\mathrm{v}}$ suit une loi en $T^{3}$ [1]. Dans un solide, considéré comme un milieu élastique continu et limité par des surfaces libres, Bolt [2] et Maa [3] ont montré que la distribution en fréquences des phonons fait intervenir, en plus du terme proportionnel à $v^{2}$, un terme proportionnel à $v$ et au rapport de l'aire de la surface du solide à son volume. Montroll [4] a montré que ce dernier terme donne, aux basses températures, une contribution à $C_{\mathrm{v}}$ proportion- nelle à $T^{2}$. Maradudin et Wallis [5], puis Dobrzynski et Leman [6] ont retrouvé cette contribution en $T^{2}$ avec des modèles de cristaux cubiques simples avec surface plates. Des mesures de $C_{\mathrm{v}}$, faites aux basses températures sur des poudres très fines de $\mathrm{NaCl}$ [7] et de $\mathrm{MgO}$ [8], semblent mettre en évidence une telle contribution, qui peut être, suivant les échantillons utilisés, jusqu'à trois fois plus grande que les prévisions théoriques [4], [5], [6]. Il semble que les valeurs de $C_{\mathrm{v}}$ mesurées soient assez sensibles à la technique utilisée pour préparer les échantillons [7].

Dobrzynski et Friedel [9] ont calculé la contribution des phonons à l'entropie des surfaces plates (100), (110) et (111) de cristaux cubiques, centrés, à faces centrées et diamant, aux hautes températures, dans l'approximation d'Einstein [10], [11] en utilisant la méthode des moments. La comparaison avec les valeurs expérimentales [12] à [15] montre que, dans ce cas, cette approximation est très valable. 
L'on sait que les surfaces cristallines peuvent présenter une certaine rugosité, à l'échelle atomique, due essentiellement à la présence de marches et de décrochements de marches [10], [16]. De tels défauts superficiels ont été observés sur différents matériaux, par exemple au moyen de la technique de décoration de leurs surfaces par l'or qui vient se placer, de façon préférentielle et en petits agrégats, le long des extrémités des marches [17] à [20]. On montre que ces défauts ont une influence sensible sur les propriétés des surfaces des points de vue de la vitesse de leur croissance, qui est plus grande que dans le cas des surfaces plates [21], et des phénomènes d'adsorption, d'évaporation, de diffusion et de catalyse superficielles [22 à 26].

Les atomes situés le long des lisières des marches et des décrochements de marche sont moins liés que les atomes d'une surface parfaitement plate. De ce fait, ils ont une plus grande liberté de mouvement, ce qui tend à augmenter l'entropie et la chaleur spécifique à volume constant des surfaces plates et à diminuer leur énergie libre. En diffraction des électrons lents (D. E. L.), la contribution de ces atomes à l'intensité diffractée par un volume de cristal avoisinant sa surface sera plus faible que la contribution des atomes situés en des régions où la surface est parfaitement plate. On peut penser que si la concentration en de tels défauts est élevée, ceux-ci peuvent perturber, de façon décelable, les propriétés vibrationnelles des surfaces.

Dans ce qui suit, on étudie les perturbations produites par des marches [100] sur quelques propriétés vibrationnelles d'un cristal avec une surface plate (001): perturbations de la densité d'états, variations de l'énergie libre $F$, de l'entropie $S$, de la chaleur spécifique à volume constant $C_{\mathrm{v}}$ et du déplacement carré moyen des atomes. Signalons l'étude commencée par l'un de nous [27], des perturbations produites par les marches sur les propriétés électroniques d'un cristal. Les calculs sont conduits au moyen de deux méthodes : la première utilise les fonctions de Green [28], associées à la méthode des déphasages généralisés introduits par de Witt [29] et Toulouse [30]. Cette méthode a été appliquée, par Dobrzynski et Leman [6], à l'étude des propriétés vibrationnelles de surfaces cristallines plates, en suivant une suggestion de Friedel et al. [31]. Elle est rigoureuse, mais implique quelques calculs numériques. La seconde méthode, très simple et analytique, utilise la méthode des moments. Elle a été utilisée pour le calcul des moyennes quadratiques des déplacements des atomes d'un cristal [32], [33]. Ces deux méthodes de calcul, très générales, sont appliquées au modèle de cristal, cubique simple, de Montroll-Potts [34], décrit au paragraphe 3 .

2. Résolution formelle par la méthode des déphasages. - Les équations du mouvement indépendantes du temps des $\mathrm{N}^{3}$ atomes d'un cristal s'écrivent, dans l'approximation harmonique, sous la forme matricielle suivante :

$$
D\left|u_{\mathrm{n}}>=\omega^{2}\right| u_{\mathrm{n}}>
$$

où $D$ est un opérateur représenté, dans une base adéquate, par une matrice, notée $D$, qui est la matrice dynamique associée au cristal étudié : les éléments de $D$ sont déterminés par les forces qui s'exercent entre les atomes. $\mid u_{\mathrm{n}}>$ est un vecteur colonne composé de $3 \mathrm{~N}^{3}$ éléments qui sont les déplacements des $\mathrm{N}^{3}$ atomes du cristal suivant les troix axes d'un repère trirectangle oxyz. $\omega$ est une fréquence angulaire de vibration.

La résolution de l'équation (2.1) est simplifiée si l'on tient compte des symétries présentées par le cristal : on peut alors se ramener à un problème unidimensionnel. On part d'un cristal cubique simple $C_{\mathrm{s}}$, semiinfini, limité par une surface (001) libre, propre et parfaitement plate, et constitué de $\mathrm{N}^{3}$ atomes identiques de masse $M$. Soit $D_{\mathrm{s}}$ la matrice dynamique qui lui est associée. On écrit $D_{\mathrm{s}}$ sous la forme :

$$
D_{\mathrm{s}}=D_{0}+V_{\mathrm{s}}
$$

où $D_{0}$ est la matrice dynamique associée au cristal parfait infini $C_{0}$ et $V_{\mathrm{s}}$ est la matrice qui représente la perturbation introduite à $C_{0}$ par sa coupure en deux cristaux semi-infinis ayant chacun une surface (001).

On obtient un cristal $C_{\mathrm{m}}$, avec deux marches parallèles à la direction de $o x(=[100])$ et éloignées d'une distance interatomique $a$, en supprimant les forces mises en jeu entre les atomes de deux rangées successives parallèles à la direction [100] (Fig. 1a). Soit $D_{\mathrm{m}}$ la
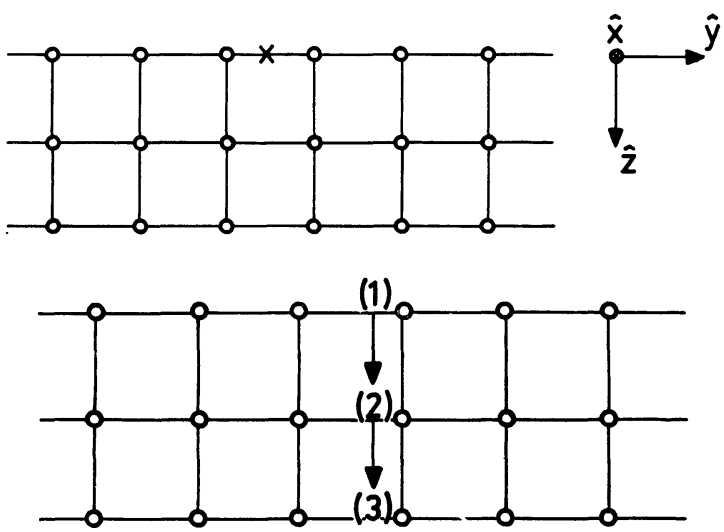

FIG. $1 a$ et $1 b$. - Modèles de cristal cubique simple avec deux marches [100] distantes respectivement de $a$ et $2 a$ ( $a=$ distance entre deux atomes premiers voisins).

matrice dynamique associée à $C_{\mathrm{m}}$. On écrit $D_{\mathrm{m}}$ sous la forme suivante :

$$
D_{\mathrm{m}}=D_{\mathrm{s}}+V_{\mathrm{m}}
$$

où $V_{\mathrm{m}}$ représente la perturbation produite sur $C_{\mathrm{s}}$ par la création des deux marches [100].

On associe à $C_{0}, C_{\mathrm{s}}$ et $C_{\mathrm{m}}$ respectivement les opérateurs fonction de Green $G_{0}^{+}, G_{\mathrm{s}}^{+}$et $G_{\mathrm{m}}^{+}$:

$$
\begin{aligned}
& G_{0}^{+}=\left(\omega^{2}-D_{0}+i \varepsilon\right)^{-1} \\
& G_{\mathrm{s}}^{+}=\left(\omega^{2}-D_{\mathrm{s}}+i \varepsilon\right)^{-1} \\
& G_{\mathrm{m}}^{+}=\left(\omega^{2}-D_{\mathrm{m}}+i \varepsilon\right)^{-1}
\end{aligned}
$$


où $\varepsilon$ est un infiniment petit positif.

$G_{\mathrm{s}}^{+}$et $G_{\mathrm{m}}^{+}$s'obtiennent respectivement à partir de $G_{0}^{+}$ et $G_{\mathrm{s}}^{+}$au moyen des relations :

$$
\begin{aligned}
& G_{\mathrm{s}}^{+}=G_{0}^{+}+G_{0}^{+} V_{\mathrm{s}} G_{\mathrm{s}}^{+} \\
& G_{\mathrm{m}}^{+}=G_{\mathrm{s}}^{+}+G_{\mathrm{s}}^{+} V_{\mathrm{m}} G_{\mathrm{m}}^{+} .
\end{aligned}
$$

On extrait toutes les informations dont on a besoin $\mathrm{du}$ $\operatorname{det}\left|I-V_{\mathrm{m}} G_{\mathrm{s}}^{+}\left(\omega^{2}+i \varepsilon\right)\right|$, après avoir exprimé $G_{\mathrm{s}}^{+}$ et $V_{\mathrm{m}}$ dans une base adéquate déterminée par l'étude des propriétés de symétrie de $C_{\mathrm{m}}$ (ponctuelles et de translation).

Ainsi les pulsations $\omega$ des états de surface perturbés par les marches des résonances et des antirésonances possibles sont les solutions de l'équation :

$$
\operatorname{Re} \operatorname{det}\left|I-V_{\mathrm{m}} G_{\mathrm{s}}^{+}\left(\omega^{2}+i \varepsilon\right)\right|=0
$$

où $\operatorname{Re} Z$ désigne la partie réelle de $Z$.

Le déphasage $\eta_{\mathrm{m}}\left(\omega^{2}\right)$, produit par la pertrubation $V_{\mathrm{m}}$ sur $C_{\mathrm{s}}$, est défini par l'égalité $[29,30]$ :

$$
\eta_{\mathrm{m}}\left(\omega^{2}\right)=-\operatorname{Arg} \operatorname{det}\left|I-V_{\mathrm{m}} G_{\mathrm{s}}^{+}\left(\omega^{2}+i \varepsilon\right)\right| .
$$

Les états de surface perturbés par les marches, s'ils existent, correspondent à une condition d'indétermination de $\eta_{\mathrm{m}}$ et la variation $\delta \rho_{\mathrm{m}}\left(\omega^{2}\right)$ de la densité des états étendus, par unité de $\omega^{2}$, est :

$$
\delta \rho_{\mathrm{m}}\left(\omega^{2}\right)=\frac{1}{\pi} \frac{\mathrm{d} \eta_{\mathrm{m}}}{\mathrm{d}\left(\omega^{2}\right)} .
$$

Dans l'approximation harmonique, on peut exprimer toute fonction thermodynamique $F$ caractérisant le cristal par une somme sur tous les états $\left(j, \mathbf{k}_{\sigma}\right)$, soit :

$$
F=\sum_{j, \mathbf{k}} f\left[\omega_{j}^{2}\left(k_{\sigma}\right)\right]
$$

où $j$ est l'indice de polarisation des ondes, de vecteur d'onde $\mathbf{k}$, qui décrivent le mouvement des $\mathrm{N}^{3}$ atomes. La variation de la densité de ces états $(j, \mathbf{k})$ due à la perturbation $V_{\mathrm{m}}$ produit sur $F$ une variation $\Delta F_{\mathrm{m}}$ que l'on peut calculer au moyen de la formule suivante [35] :

$$
\Delta F_{\mathrm{m}}=-\frac{1}{\pi} \int \mathrm{d} \omega \frac{\partial f}{\partial \omega} \eta_{\mathrm{m}} .
$$

3. Propriétés du modèle de cristal de MontrollPotts [34]. - C'est un modèle de cristal cubique simple constitué de $\mathrm{N}^{3}$ atomes identiques de masses $M$. Les interactions entre atomes, limitées aux premiers voisins, sont supposées harmoniques. L'énergie potentielle du cristal s'écrit, dans ce modèle :

$$
U=U_{0}+\frac{K}{2} \sum_{\mathbf{l}, \mathbf{l}^{\prime}, \sigma}\left[u^{\sigma}(\mathbf{l})-u^{\sigma}\left(\mathbf{l}+\mathbf{l}^{\prime}\right)\right]^{2}
$$

où $U_{0}$ est l'énergie potentielle du cristal lorsque tous les atomes sont à l'état d'équilibre, $K$ est la constante de force centrale entre premiers voisins, $u^{\sigma}(\mathbf{l})$ désigne la composante suivant $\sigma=x, y$ ou $z$ du déplacement, par rapport à sa position d'équilibre, de l'atome situé au site $\mathbf{l}(h, l, n) ;\left(\mathbf{l}+\mathbf{l}^{\prime}\right)$ désigne les sites premiers voisins du site $\mathbf{l}$.

La relation de dispersion des ondes élastiques, de vecteur d'onde $\mathbf{k}$, trois fois dégénérées dans ce modèle, s'écrit :

$\omega_{j}^{2}\left(k_{\sigma}\right)=2 \frac{K}{M}\left(3-\cos \varphi_{x}-\cos \varphi_{y}-\cos \varphi_{z}\right)$

où $j=1,2,3, \varphi_{\sigma}=a k_{\sigma}$ avec $a=$ distance entre atomes premiers voisins.

La pulsation maximale est égale à :

$$
\omega_{\mathrm{m}}=\sqrt{12 \frac{K}{M}} .
$$

Les vecteurs propres de $D_{0}$ peuvent être pris égaux à :

$$
\mathbf{u}(\mathbf{l})=\exp (i \mathbf{k} \cdot \mathbf{l}) \hat{\sigma}
$$

où $\hat{\sigma}$ désigne le vecteur unitaire des directions $x(=[100]), y(=[010])$ ou $z(=[001])$.

Il faut noter que l'énergie potentielle, associée à ce modèle, n'est pas invariante pour des rotations infinitésimales de l'ensemble du cristal. Or cette invariance est nécessaire pour que les pulsations des ondes soient les mêmes que celles que donne la théorie de l'élasticité [36]. Bien que le modèle utilisé ici ne puisse conduire à un tel accord, il donne de bonnes estimations de la contribution des phonons aux fonctions thermodynamiques des surfaces [6], [37]. Le cristal $C_{\mathrm{m}}$ présente une symétrie de translation dans la direction de $x(=[100])$ seulement, ce qui implique que les éléments des matrices représentant les opérateurs fonction de Green, définis par (2.4), (2.5) et (2.6), ne dépendent que de $\varphi_{x}$ : les éléments de la matrice représentant $G_{0}^{+}$ s'écrivent :

$$
\begin{aligned}
& G_{0}^{+\sigma \sigma^{\prime}}(E ; l ; n)= \\
& =\frac{\delta_{\sigma \sigma^{\prime}}}{(2 \pi)^{2}} \frac{M}{2 K} \int_{-\pi}^{\pi} \mathrm{d} \varphi_{y} \int_{-\pi}^{\pi} \mathrm{d} \varphi_{z} \frac{\cos \left(l \varphi_{y}\right) \cos \left(n \varphi_{z}\right)}{E+\cos \varphi_{y}+\cos \varphi_{z}+\imath \varepsilon}
\end{aligned}
$$

où

$$
E=\frac{M}{2 K} \omega^{2}-3+\cos \varphi_{x}
$$

$\sigma, \sigma^{\prime}=x, y$ ou $z ; \delta_{\sigma \sigma^{\prime}}$ représente le symbole de Kronecker.

Les éléments de la matrice représentant $G_{\mathrm{s}}^{+}$, qui intervient dans les équations (2.9) et (2.12), sont obtenus à partir des éléments de $G_{0}^{+}$par la relation suivante [36]:

$$
\begin{aligned}
& G_{\mathrm{s}}^{+}\left(E ; l ; n, n^{\prime}\right)= \\
& \quad=G_{0}^{+}\left(E ; l ; n-n^{\prime}\right)+G_{0}^{+}\left(E ; l ; n+n^{\prime}-1\right) .
\end{aligned}
$$

4. Perturbations produites par des marches [100] sur les propriétés dynamiques du modèle de cristal de 
Montroll-Potts. - Les éléments de la matrice $V_{\mathrm{m}}$, définie au paragraphe 2, équation (2.3), s'écrivent:

$$
\begin{aligned}
& V_{\mathrm{m}}^{\sigma \sigma^{\prime}}\left(\varphi_{x} ; 1, \mathrm{l}^{\prime}\right)= \\
& =\delta_{\sigma \sigma^{\prime}} \frac{K}{M}\left(\delta_{n 1} \delta_{n^{\prime} 1} \delta_{l 0} \delta_{l^{\prime} 1}+\delta_{n 1} \delta_{n^{\prime} 1} \delta_{l 1} \delta_{l^{\prime} 0}-\right. \\
& \left.\quad-\delta_{n 1} \delta_{n^{\prime} 1} \delta_{l 0} \delta_{l^{\prime} 0}-\delta_{n 1} \delta_{n^{\prime} 1} \delta_{l 1} \delta_{l^{\prime} 1}\right)
\end{aligned}
$$

La connaissance de $G_{\mathrm{s}}^{+}$et $V_{\mathrm{m}}$ permet de calculer le $\operatorname{det}\left|I-V_{\mathrm{m}} G_{\mathrm{s}}^{+}\right|$. La résolution de l'équation (2.9) et l'étude du déphasage $\eta_{\mathrm{m}}$, défini par l'équation (2.10), permettent de déterminer les pulsations $\omega$ des états de surfaces perturbées par les marches, des résonances et des antirésonances possibles [31], [38] : les états de surface perturbés par les marches, s'ils existent, correspondent à une indétermination de $\eta_{\mathrm{m}}$. A l'intérieur des bandes des états étendus, $\eta_{\mathrm{m}}$ passe par la valeur $\pi / 2$, avec $\delta \rho_{\mathrm{m}}\left(\omega^{2}\right)>0$ (augmentation de la densité d'états : cf. éq. (2.11)) à la pulsation correspondant à une résonance possible, et avec $\delta \rho_{\mathrm{m}}\left(\omega^{2}\right)<0$ (diminution de la densité d'états) à la pulsation correspondant à une antirésonance possible.

L'on sait que le modèle de Montroll-Potts ne donne pas des états de surface. Il ne permet donc pas d'étudier l'effet des marches sur ces états. On peut cependant dire, de façon générale, que la présence de défauts peu étendus à la surface d'un cristal (monocouche adsorbée, marches peu étendues) ne modifie pas la vitesse des ondes de Rayleigh, mais agit sur les pulsations des états de surface.

On a résolu l'équation (2.9), numériquement, en prenant $E$ comme inconnue. Notons que l'intérieur des bandes des états étendus, à $\varphi_{x}$ donné, correspond à : $-2<E<2$. A l'intérieur de ces bandes, l'équation (2.9) a deux racines :

$$
\begin{aligned}
E_{\mathrm{R}} & \simeq-0,35 \\
E_{\mathrm{AR}} & \simeq-0,05 .
\end{aligned}
$$

On a représenté les relations de dispersion correspondantes, $\omega^{2}=\omega^{2}\left(\varphi_{x}\right)$ sur la figure 2 (courbes $R$ et AR). Sur cette même figure, on a représenté la bande des états étendus, à $\varphi_{x}$ donné. L'étude de la variation de $\eta_{\mathrm{m}}$ en fonction de $E$ montre que le long des courbes $\mathrm{R}$ et $\mathrm{AR}$ on a respectivement une augmentation et une diminution de la densité d'états : la présence des marches abaisse les pulsations des ondes élastiques en volume.

La condition pour laquelle une résonance a un aspect aigu et bien défini dans la densité d'états est obtenue à partir de l'expression de la variation de la densité d'états au voisinage de cette résonance. On a, en utilisant (2.11) et (2.10) :

$$
\delta \rho_{\mathrm{m}}(E)=-\frac{3}{\pi} \frac{\mathrm{d}}{\mathrm{d} E}\left[\operatorname{Arctg} \frac{J(E)}{R(E)}\right]
$$

où $R(E)$ et $J(E)$ désignent respectivement les parties réeilie et imaginaire du det $\left|I-V_{\mathrm{m}} G_{\mathrm{s}}^{+}(E+i \varepsilon)\right|$.

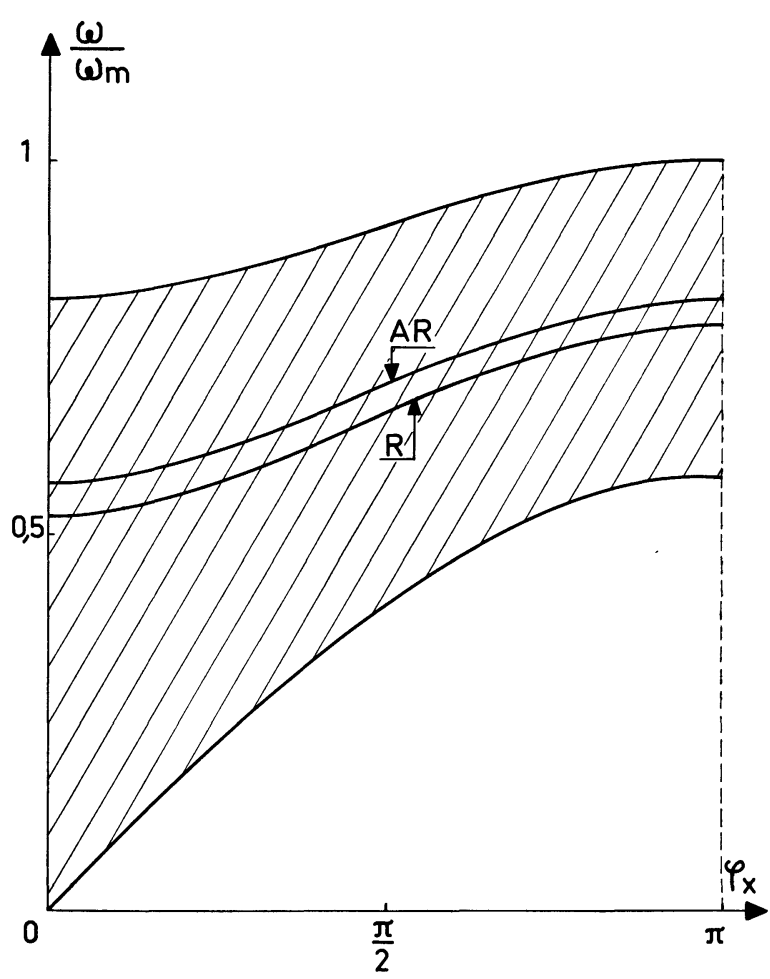

Fig. 2. - Modèle de cristal, cubique simple, de MontrollPotts. La région hachurée représente la bande des pulsations $\omega$ des ondes de volume (à $\varphi_{x}$ donné) qui se propagent parallèlement au plan (001). Les courbes R (augmentation de la densité des ondes en volume) et AR (diminution de cette densité) représentent les solutions $\omega^{2}=\omega^{2}\left(\varphi_{x}\right)$ de l'équation (2.9), dans le cas de la figure $1 a$.

Pour $E \simeq E_{\mathrm{R}}, \delta \rho_{\mathrm{m}}(E)$ est donnée par la formule approchée :

$$
\delta \rho_{\mathrm{m}}(E) \simeq \frac{3}{\pi} \frac{\Gamma}{\left(E-E_{\mathrm{R}}\right)^{2}+\Gamma^{2}}
$$

où

$$
\Gamma=J(E) /\left(\frac{\partial R}{\partial E}\right)_{E=E_{\mathrm{R}}} .
$$

La résonance est bien définie si : $|\Gamma| E_{\mathrm{R}} \mid \ll 1$.

Pour la résonance trouvée, on a $:|\Gamma| E_{\mathrm{R}} \mid \simeq 7$ ce qui montre qu'elle est mal définie.

5. Variations des fonctions thermodynamiques d'une surface plate (001) dues à la présence de deux marches [100], distantes de a. - 5.1 MÉTHODE, EXACTE, DES DÉPHASAGES. - Ces variations sont calculées, numériquement, au moyen de la formule (2.13), en fonction de la température $T$ du cristal. Le déphasage à utiliser est le déphasage total donné par l'égalité :

$\eta_{\mathrm{m}}\left(\omega^{2}\right)=\frac{3 N}{2 \pi} \int_{-\pi}^{\pi} \mathrm{d} \varphi_{x} \eta_{\mathrm{m}}\left(E=\frac{M}{2 K} \omega^{2}-3+\cos \varphi_{x}\right)$

où $\omega$ est une pulsation des ondes élastiques en volume ; $N$ est le nombre d'atomes le long d'une marche et le facteur 3 tient compte de la triple dégénérescence des 
phonons. Sur la figure 3, on a représenté la courbe de variation de $\eta_{\mathrm{m}}$, par atome de coin de marche, en fonction de la pulsation $\omega$.

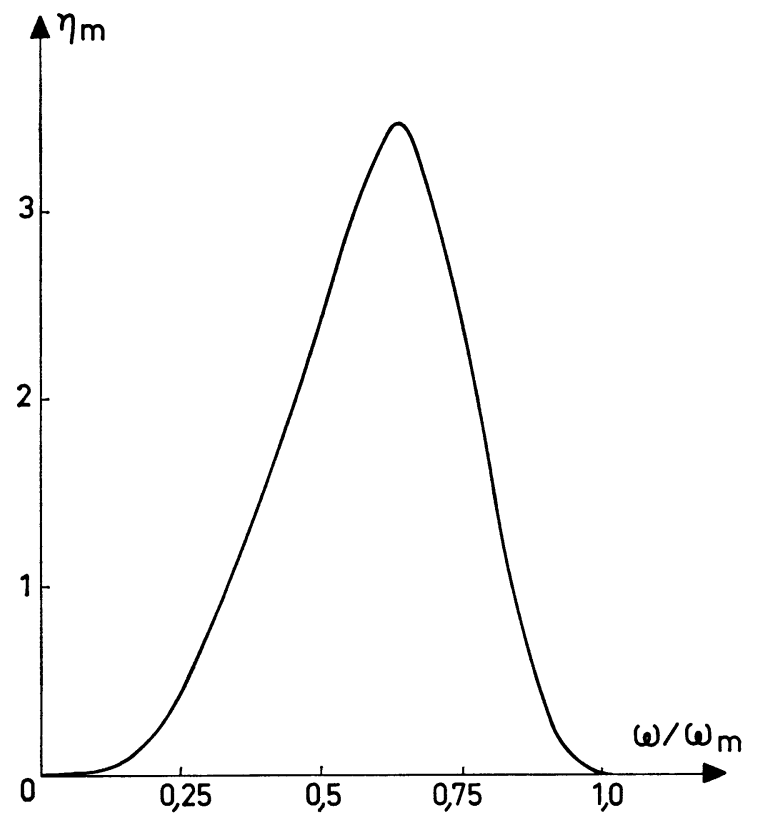

Fig. 3. - Modèle de cristal, cubique simple, de MontrollPotts. Courbe de variation du déphasage total $\eta_{\mathrm{m}}(\omega)$ introduit, par atome au coin d'une marche (cf. Fig. 1a), aux ondes de volume, de pulsation $\omega$.

Si $F$ représente respectivement l'énergie libre $F$, l'entropie $S$ et la chaleur spécifique à volume constant $C_{\mathrm{v}}$, la fonction $f$, définie par (2.12), s'écrit sous les formes suivantes :

$$
\begin{aligned}
f_{F} & =k_{\mathrm{B}} T\left[\frac{x}{2}+\ln \left(1-\mathrm{e}^{-x}\right)\right] \\
f_{S} & =k_{\mathrm{B}}\left[\frac{x}{\mathrm{e}^{x}-1}-\ln \left(1-\mathrm{e}^{-x}\right)\right] \\
f_{C_{\mathrm{v}}} & =k_{\mathrm{B}} \frac{x^{2} \mathrm{e}^{x}}{\left(\mathrm{e}^{x}-1\right)^{2}}
\end{aligned}
$$

avec

$$
x=\frac{\omega_{j}(\mathbf{k})}{\omega_{\mathrm{m}}} \frac{T_{\mathrm{D}}}{T}
$$

où $T_{\mathrm{D}}$ désigne la température de Debye, en volume, telle que :

$$
k_{\mathrm{B}} T_{\mathrm{D}}=\hbar \omega_{\mathrm{m}}
$$

avec $k_{\mathrm{B}}=$ constante de Boltzmann, $2 \pi \hbar=$ constante de Planck, $\omega_{\mathrm{m}}=$ pulsation maximale des ondes élastiques en volume, donnée par $\left(3.2^{\prime}\right)$.

Les figures 4,5 et 6 représentent, respectivement, les courbes $-\Delta F_{\mathrm{m}}(T), \Delta S_{\mathrm{m}}(T)$ et $\Delta C_{\mathrm{vm}}(T)$, par atome de coin de marche. Les résultats obtenus se comprennent, qualitativement, d'un point de vue intuitif : en effet, un atome situé au coin d'une marche a, de façon schématique, une liaison de moins qu'un atome situé sur une surface parfaitement plate. Il se trouve donc dans un

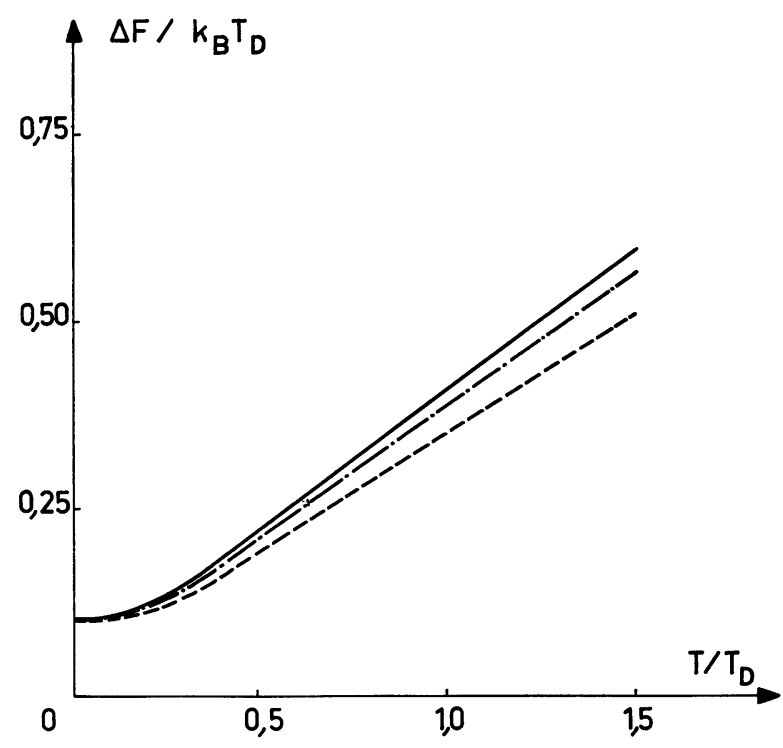

FIG. 4. - Modèle de cristal, cubique simple, de MontrollPotts. Courbes représentant la perturbation (changée de signe) introduite, par un atome au coin d'une marche (cf. Fig. 1a), à l'énergie libre, de vibration, d'une surface (001) plate, en fonction de la température $T$.

Calcul exact par la méthode des déphasages.

- Approximation d'Einstein.

-. - Approximation d'Einstein + premier terme correctif.

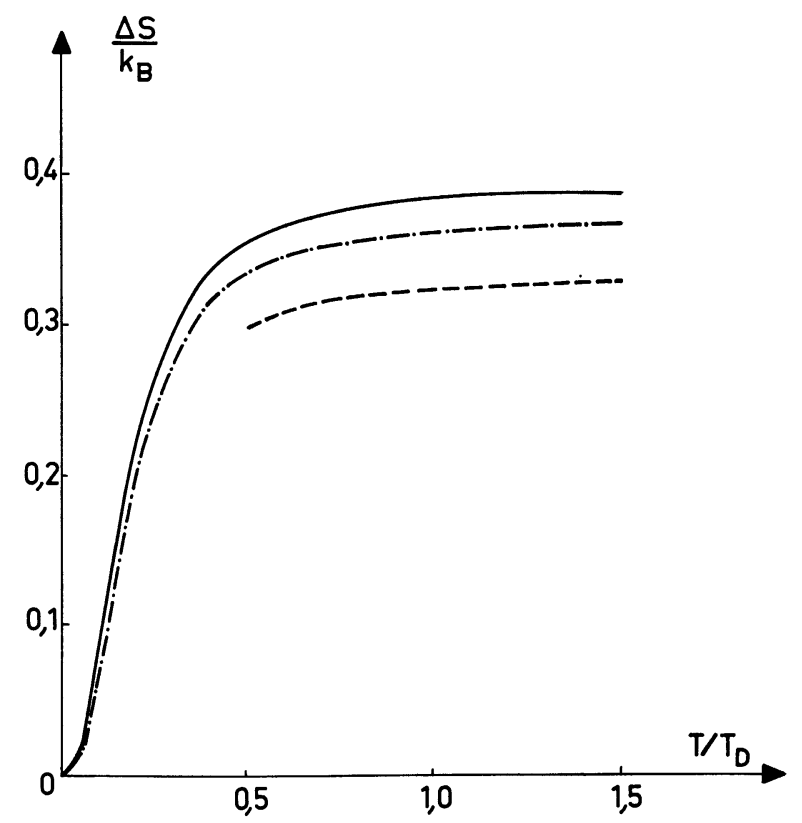

FIG. 5. - Modèle de cristal, cubique simple, de MontrollPotts. Courbes représentant la perturbation introduite, par un atome au coin d'une marche (cf. Fig. 1a) à l'entropie $S$, de vibration, d'une surface (001) plate, en fonction de la température $T$.

— Calcul exact par la méthode des déphasages.

- - Approximation d'Einstein.

-. - Approximation d'Einstein + premier terme correctif.

état de plus grande liberté de mouvement, ce qui entraîne un accroissement de l'entropie $S$ et de la chaleur spécifique $C_{\mathrm{v}}$ de la surface et en conséquence une diminution de son énergie libre $F$. 


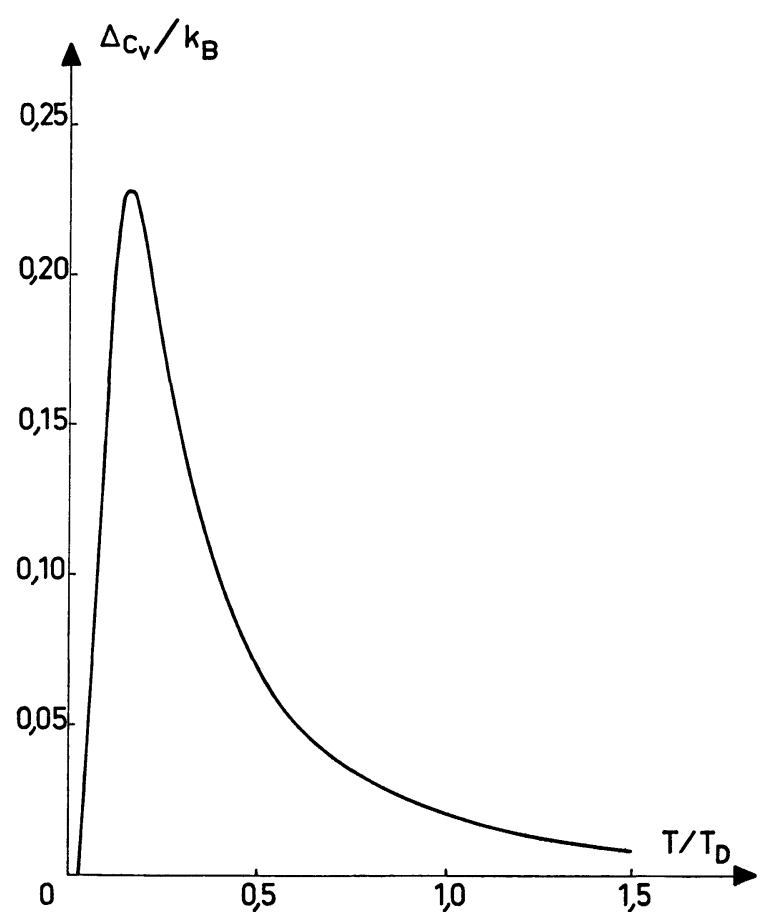

FIG. 6. - Modèle de cristal, cubique simple, de MontrollPotts. Courbe représentant la perturbation introduite, par un atome au coin d'une marche (cf. Fig. 1a), à la chaleur spécifique $C_{\mathrm{v}}$, de vibration, d'une surface (001) plate, en fonction de la température $T$ (méthode des déphasages).

5.2 MÉTHODE APPROCHÉE, BASÉE SUR LA MÉTHODE DES MOMENTS. - On calcule ces variations, dans ce qui suit, par une méthode très simple et analytique, basée sur la méthode des moments, et qui a été déjà utilisée pour le calcul des moyennes quadratiques des déplacements des atomes d'un cristal [32], [33].

On part de l'expression de l'énergie libre $F$ d'un cristal en fonction de la matrice dynamique $D$ qui lui est associée [39] :

$$
F=F_{0}+k_{\mathrm{B}} T \operatorname{tr}\left\{\ln \left[2 \operatorname{sh}\left(\frac{\hbar D^{1 / 2}}{2 k_{\mathrm{B}} T}\right)\right]\right\}
$$

où $F_{0}$ est l'énergie libre lorsque tous les atomes sont à l'état d'équilibre.

$$
\operatorname{tr}(A)=\text { trace de la matrice } A .
$$

On écrit, comme Salter :

$$
D=d+R
$$

où $d$ est la partie diagonale de $D$ et $R$ la matrice $D$ - $d$.

On développe $F$, en série de Taylor $(*)$, au voisinage de $F(d)$.

On obtient ainsi :

$$
F=F_{0}+F(d)+F\left(R^{2}\right)+\cdots
$$

(*) Dans tous les cas où les matières $d$ et $R$ ne commutent pas, il est plus exact d'utiliser le développement donné par M. R. Schafroth (Helv. Phys. Acta, 1951, 24, 645). Mais nous avons vérifié que dans notre cas les différences entre les résultats numériques donnés par les deux développements sont inférieures à $2 \%$. où

$$
F(d)=k_{\mathrm{B}} T \operatorname{tr}\left\{\ln \left[2 \operatorname{sh}\left(\frac{\hbar d^{1 / 2}}{2 k_{\mathrm{B}} T}\right)\right]\right\}
$$

et

$$
\begin{aligned}
F\left(R^{2}\right)=-\frac{\hbar}{16} \operatorname{tr}\left[R ^ { 2 } \left(d^{-3 / 2} \operatorname{coth} A+\right.\right. \\
\left.\left.\quad+\frac{\hbar}{2 k_{\mathrm{B}} T} d^{-1} \operatorname{sh}^{-2} A\right)\right]
\end{aligned}
$$

avec

$$
A=\frac{\hbar d^{1 / 2}}{2 k_{\mathrm{B}} T}
$$

$F(d)$ représente l'expression de l'énergie libre dans l'approximation d'Einstein.

Soient :

$$
D_{\mathrm{s}}=d_{\mathrm{s}}+R_{\mathrm{s}}
$$

et

$$
D_{\mathrm{m}}=d_{\mathrm{m}}+R_{\mathrm{m}}
$$

les matrices dynamiques associées respectivement aux cristaux $C_{\mathrm{s}}$ et $C_{\mathrm{m}}$, définis au paragraphe 2 . On obtient, compte tenu de (5.9), (5.10) et (5.11) :

$\Delta F_{\mathrm{m}}=F_{\mathrm{m}}-F_{\mathrm{s}}=\Delta F_{\mathrm{m}}(d)+\Delta F_{\mathrm{m}}\left(R^{2}\right)+\cdots$

où

$$
\Delta F_{\mathrm{m}}(d)=F\left(d_{\mathrm{m}}\right)-F\left(d_{\mathrm{s}}\right)
$$

et

$$
\Delta F_{\mathrm{m}}\left(R^{2}\right)=F\left(R_{\mathrm{m}}^{2}\right)-F\left(R_{\mathrm{s}}^{2}\right) .
$$

Les variations d'entropie $S$ et de chaleur spécifique à volume constant $C_{\mathrm{v}}$ s'obtiennent au moyen des formules suivantes :

$$
\begin{aligned}
& \Delta S_{\mathrm{m}}=-\frac{\partial}{\partial T}\left(\Delta F_{\mathrm{m}}\right)_{\mathrm{v}} \\
& \Delta C_{\mathrm{vm}}=\left(\frac{\partial}{\partial T}\right)_{\mathrm{v}}\left(\Delta F_{\mathrm{m}}+T \Delta S_{\mathrm{m}}\right) .
\end{aligned}
$$

Les traces des matrices intervenant dans les expressions précédentes sont calculées par une méthode due à Cyrot-Lackmann [40].

5.2.1 Approximation d'Einstein. - On sait que cette approximation permet d'obtenir de bonnes valeurs approchées de l'entropie de surface, dans le domaine des hautes températures par rapport à la température de Debye $T_{\mathrm{D}}$ [9]. Dans le domaine des basses températures $\left(T \ll T_{\mathrm{D}}\right)$, elle donne de mauvais résultats pour l'entropie $S$ et la chaleur spécifique $C_{\mathrm{v}}$ : en effet, la contribution des phonons à une fonction thermodynamique $F$ s'écrit :

$$
F(T)=3 r N^{3} \int_{0}^{\omega_{\mathbf{m}}} \mathrm{d} \omega f(\omega, T) \rho(\omega)
$$

où $\rho(\omega)=$ la densité d'états, $f(\omega, T)=$ la fonction définie par (2.12) et donnée par les formules (5.2), (5.3) et (5.4) respectivement si $F$ désigne l'énergie libre $F$, l'entropie $S$ et la chaleur spécifique $C_{\mathrm{v}}, r=$ le 
nombre d'atomes par cellule élémentaire, $N^{3}=$ le nombre de cellules élémentaires dans le cristal.

La connaissance, détaillée, de $\rho(\omega)$ permet de calculer $F(T)$, exactement, pour toute température. Dans l'approximation d'Einstein on suppose que les atomes du cristal vibrent à la même pulsation $\omega_{\mathrm{E}}=\sqrt{6 K / M}$. Ceci revient à remplacer, dans (5.19), $\rho(\omega)$ par une fonction «delta » centrée au barycentre de $\rho(\omega)$. Les courbes de variation de $f_{S}$ et $f_{C_{v}}$ en fonction de $\omega$ (Fig. 7 et 8) montrent que ces fonctions approchent leurs valeurs maximales à des pulsations d'autant plus éloignées de la pulsation d'Einstein $\omega_{\mathrm{E}}$ que la température $T$

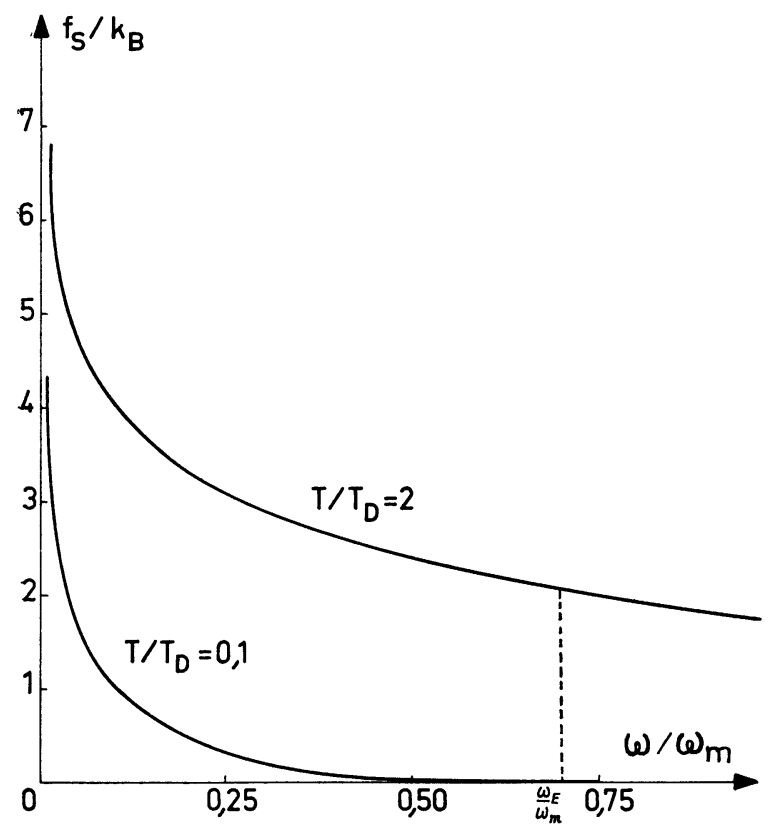

FIG. 7. - Courbes de variation de $f_{\mathrm{s}}$ (en unités $k_{\mathrm{B}}$ ) en fonction de $\omega / \omega_{\mathrm{m}}$ (cf. formule (5.3)).

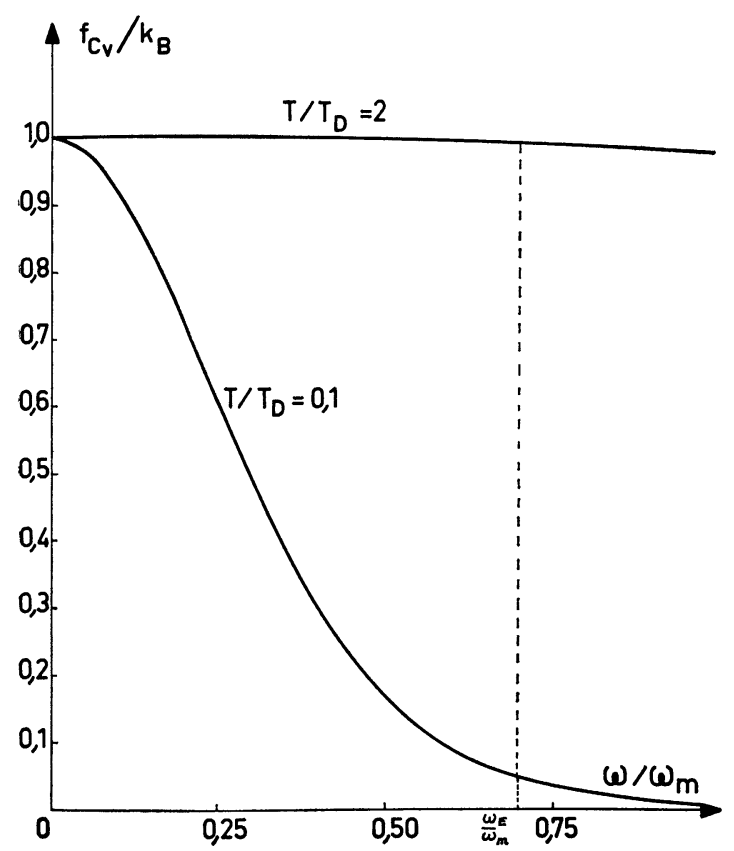

FIG. 8. - Courbes de variation de $f_{C \mathrm{v}}$ (en unités $k_{\mathrm{B}}$ ) en fonction de $\omega / \omega_{\mathrm{m}}$ (cf. formule (5.4)). est plus faible. C'est pour cette raison que l'approximation d'Einstein ne peut donner de bonnes valeurs approchées de $S$ et $C_{\mathrm{v}}$ aux basses températures. Par contre, les courbes de variation de $f_{F}$ en fonction de $\omega$ (Fig. 9)

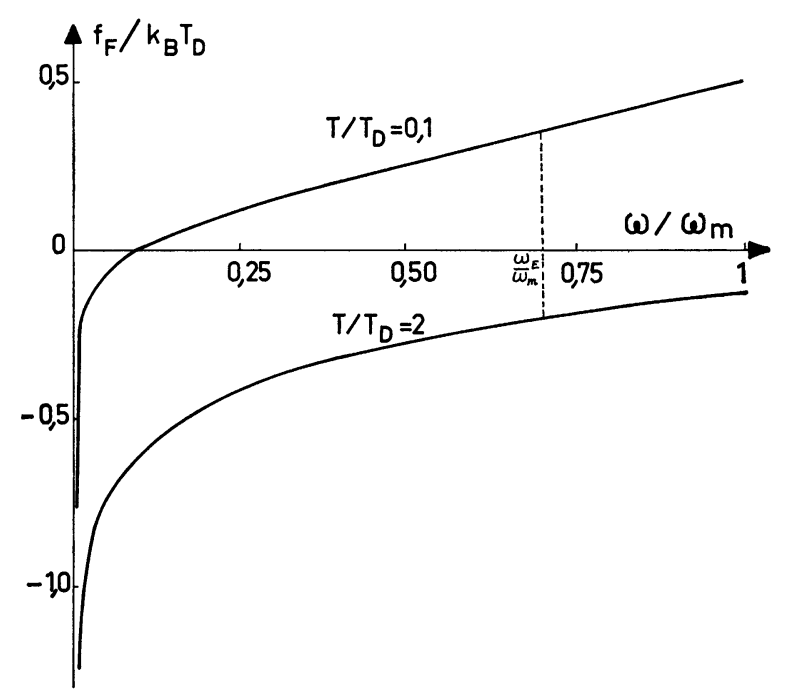

Fig. 9. - Courbes de variation de $f_{F}$ (en unités $k_{\mathrm{B}} T_{\mathrm{D}}$ ) en fonction de $\omega / \omega_{\mathrm{m}}$ (cf. formule (5.2)). Pour la courbe correspondant à $T / T_{\mathrm{D}}=2$, les ordonnées doivent être multipliées par 10 .

montrent que la partie de $f_{F}$ qui donne les contributions les plus importantes à l'intégrale $(5.19)$ correspond à un intervalle de variation de $\omega$ d'autant plus voisin de $\omega_{\mathrm{E}}$ que $T$ est plus faible. Il semble donc que l'on doit s'attendre à ce que l'approximation d'Einstein donne des valeurs de l'énergie libre qui approchent les valeurs calculées par la méthode exacte des déphasages d'autant mieux que la température est plus basse.

5.2.2 Précisions des résultats obtenus, par rapport au calcul exact par la méthode des déphasages. - On a représenté, sur les figures 4,5 et 6 , respectivement les courbes de variation, en fonction de $T$, de $-\Delta F_{\mathrm{m}}$, $\Delta S_{\mathrm{m}}$ et $\Delta C_{\mathrm{v}}$ données par les expressions (5.14), (5.17) et (5.18), limitées au premier terme correctif en $R^{2}$.

Pour l'énergie libre $F$, l'approximation d'Einstein donne une précision de $4 \%$ et $15 \%$ respectivement pour $T / T_{\mathrm{D}}=0,1$ et 2 . Les précisions correspondantes, lorsque l'on tient compte du premier terme correctif en $R^{2}$, sont de $1 \%$ et $5 \%$.

Pour l'entropie $S$, l'approximation d'Einstein donne une précision de $15 \%$ pour $T / T_{\mathrm{D}}=1$. En tenant compte du premier terme correctif, on obtient une précision de $9 \%$ et $6 \%$ respectivement pour $T / T_{\mathrm{D}}=0,2$ et 1 .

Pour la chaleur spécifique $C_{\mathrm{v}}$, l'approximation d'Einstein donne une précision de $1 \%$ pour $T / T_{\mathrm{D}}=1$. En tenant compte du premier terme correctif, la précision est de $13 \%$ et $1 \%$ respectivement pour

$$
\frac{T}{T_{\mathrm{D}}}=0,1 \text { et } 0,2 \text {. }
$$


6. Influence de la distance entre les deux marches [100] sur les fonctions thermodynamiques. - On va estimer l'ordre de grandeur des variations des fonctions thermodynamiques lorsque la distance entre les deux marches augmente. Ces variations seront certainement très faibles étant donné la nature des interactions limitées, dans le modèle utilisé, aux atomes premiers voisins seulement.

On part du modèle où les deux marches sont distantes de $a$ (Fig. 1a). On obtient un modèle avec marches distantes de $2 a$ en affaissant d'un cran une rangée d'atomes (1), comme l'indique la flèche sur la figure $1 b$. Les atomes qui étaient en (2) sont maintenant un cran plus bas. Le nombre total d'atomes étant ainsi conservé, l'expression de la variation d'entropie, dans le domaine des hautes températures, s'écrit [9] :

$$
\Delta S_{\mathrm{m}}=\Delta S_{\mathrm{m}}(d)+k_{\mathrm{B}} \sum_{n=1}^{\infty}\left[K n+0\left(\frac{1}{T^{2 n}}\right)\right]
$$

où

$\Delta S_{\mathrm{m}}(d)=$ variation d'entropie dans l'approximation d'Einstein.

$$
\begin{gathered}
K n=\frac{(-1)^{n}}{2 n} \operatorname{tr}\left\{\left[\left(d_{\mathrm{m}}^{-1} R_{\mathrm{m}}\right)^{n}-\left(d_{\mathrm{s}}^{-1} R_{\mathrm{s}}\right)^{n}\right]\right\} \\
0\left(\frac{1}{T^{2 n}}\right)=(-1)^{n-1} \frac{2 n-1}{2 n(2 n) !} \Delta \mu_{2 n} B_{2 n}\left(\frac{\hbar}{k_{\mathrm{B}} T}\right)^{2 n}
\end{gathered}
$$

avec

$$
\Delta \mu_{2 n}=\operatorname{tr}\left(D_{\mathrm{m}}^{n}-D_{\mathrm{s}}^{n}\right)
$$

$B_{2 n}=$ nombre de Bernouilli.

$\Delta S_{\mathrm{m}}(d)$ ne varie pas lorsque la distance entre les deux marches passe de $a$ à $2 a$, soit :

$$
\Delta S_{\mathrm{m}}(d) \simeq 0,335 k_{\mathrm{B}} .
$$

En limitant l'expression (7.1) aux premiers termes correctifs $K 2$ et $1 / T^{2}$, on trouve, pour l'entropie par atome de coin de marche :

Pour une distance entre marches $=a$ :

$\Delta S_{\mathrm{m}}^{a} \simeq k_{\mathrm{B}}\left[0,335+0,031-\frac{1}{480}\left(\frac{T_{\mathrm{D}}}{T}\right)^{2}+\cdots\right]$.

Pour une distance entre marches $=2 a$ :

$\Delta S_{\mathrm{m}}^{2 a} \simeq k_{\mathrm{B}}\left[0,335+0,001-\frac{1}{480}\left(\frac{T_{\mathrm{D}}}{T}\right)^{2}+\cdots\right]$.

Ainsi lorsque la distance entre les deux marches augmente, les fonctions thermodynamiques varient par des termes correctifs d'ordre de plus en plus élevé, c'est-à-dire par des quantités de plus en plus petites. La faible différence entre $\Delta S_{\mathrm{m}}^{a}$ et $\Delta S_{\mathrm{m}}^{2 a}$ peut s'expliquer par la nature des interactions limitées aux premiers voisins seulement.

7. Moyenne quadratique du déplacement d'un atome situé au coin d'une marche. - La moyenne quadratique du déplacement, dans la direction $\sigma(=x, y$ ou $z)$, d'un atome, de masse $M_{1}$, se trouvant au site 1 , s'écrit [32] :

$\left\langle u_{1 \sigma}^{2}(d+R)>=\left\langle u_{1 \sigma}^{2}(d)\right\rangle+F\left(R^{2}\right)+\cdots\right.$

où

$$
\begin{aligned}
& <u_{\mathbf{l} \sigma}^{2}(d)>=\frac{\hbar}{2 M_{1}}\left[d^{-1 / 2} \times \operatorname{coth}\left(\frac{\hbar d^{1 / 2}}{2 k_{\mathrm{B}} T}\right)\right]_{\mathbf{l} \sigma, \mathbf{l} \sigma} \\
& F\left(R^{2}\right)=\frac{\hbar}{16 M_{\mathbf{l}}}\left(R^{2}\right)_{\mathbf{l} \sigma, \mathbf{l} \sigma}\left(3 d^{-5 / 2} \operatorname{coth} A+\right. \\
& +\frac{3}{2} \frac{\hbar}{k_{\mathrm{B}} T} d^{-2} \times \operatorname{sh}^{-2} A \\
& \left.+\frac{1}{2}\left(\frac{\hbar}{2 k_{\mathrm{B}} T}\right)^{2} d^{-3 / 2} \operatorname{ch} A \operatorname{sh}^{-3} A\right)_{\mathbf{l} \sigma, \mathbf{l} \sigma}
\end{aligned}
$$

où $A$ a été définie en $\left(5.11^{\prime}\right)$.

$\left\langle u_{1 \sigma}^{2}(d)\right\rangle$ représente l'expression de $\left\langle u^{2}\right\rangle$ dans l'approximation d'Einstein.

On a calculé $\left\langle u^{2}\right\rangle$ pour un atome situé au coin d'une marche, en fonction de $T$, en limitant le développement $(*)(7.1)$ au premier terme correctif en $R^{2}$. Les résultats obtenus, représentés sur la figure 10, sont relatifs aux directions de vibration aussi bien normales que parallèles à la surface (001), puisque le modèle de cristal utilisé ne permet pas de mettre en

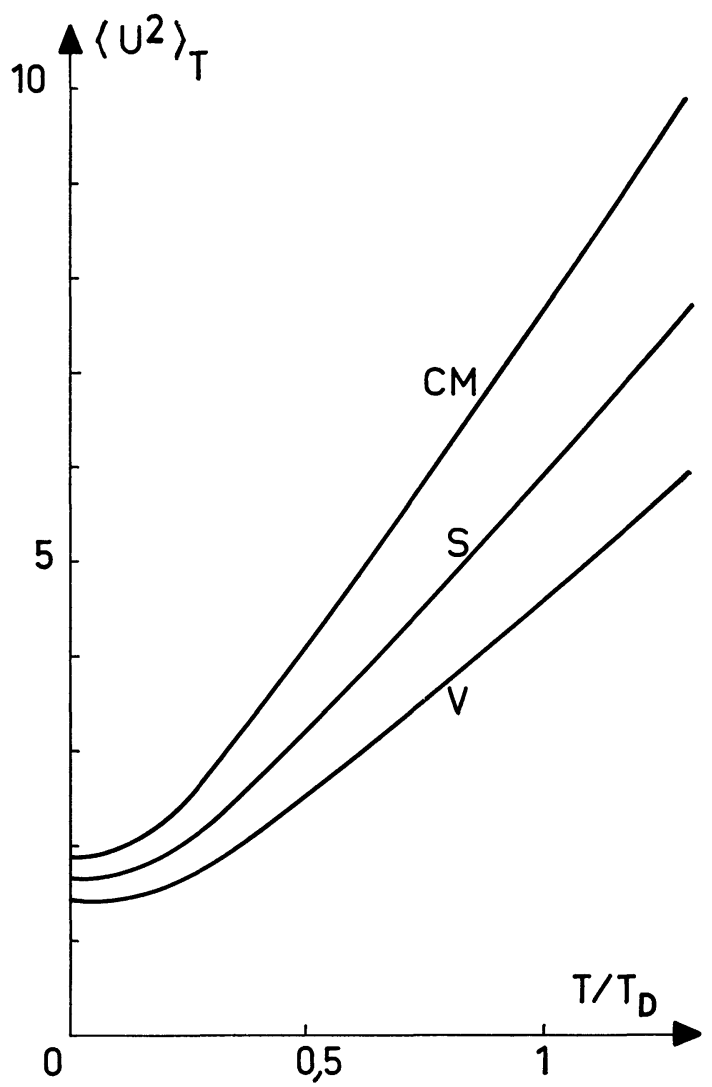

Fig. 10. - Modèle de cristal, cubique simple, de MontrollPotts. Courbes de variation, en fonction de la température $T$, de la moyenne quadratique du déplacement (en unités $\left.k_{\mathrm{B}} T_{\mathrm{D}} / 24 K\right)$ d'un atome situé en volume $(V)$, sur une surface (001) plate $(S)$, et au coin d'une marche [100] (C. M.). 
évidence une anisotropie des vibrations. Sur la même figure, dans un but de comparaison, on a porté les variations de la moyenne quadratique du déplacement d'un atome de surface plane (001) et de volume, en fonction de $T$. Pour $T=T_{\mathrm{D}}$ par exemple, les rapports des moyennes quadratiques de déplacement d'un atome au coin d'une marche à celui d'un atome de surface et de volume sont respectivement de 1,3 et 1,7. Qualitativement, ces résultats s'interprètent par le fait qu'un atome au coin d'une marche est moins lié qu'un atome de surface ou de volume. Il a donc une plus grande amplitude de vibration. De ce fait, sa contribution à l'intensité diffractée, dans des expériences de diffraction d'électrons lents, sera plus faible.

Conclusion. - Les résultats obtenus donnent l'ordre de grandeur de l'effet des marches sur quelques propriétés vibrationnelles des surfaces cristallines : par atome au coin d'une marche, on trouve une augmentation de l'entropie $S \simeq 0,39 k_{\mathrm{B}}$ à $T / T_{\mathrm{D}}=1,5$, de la chaleur spécifique $C_{\mathrm{v}} \simeq 0,23 k_{\mathrm{B}}$ à $T / T_{\mathrm{D}}=0,16$, diminution de l'énergie libre $F \simeq 0,6 k_{\mathrm{B}} T_{\mathrm{D}}$ à $T / T_{\mathrm{D}}=1,5$. Le rapport des moyennes quadratiques du déplacement d'un atome au coin d'une marche à celui d'un atome sur une surface (001) plate, perpendiculairement à cette surface, est $\simeq 1,3$, aux températures comparables à la température de Debye $T_{\mathbf{D}}$.

Bien que la contribution électronique à l'énergie libre $F$ soit la plus importante, aux basses températures, il peut être intéressant de signaler qu'à ces basses températures, l'approximation d'Einstein permet d'obtenir de bonnes valeurs approchées de la contribution à $F$ due aux phonons.

Remerciements. - Nous remercions le Professeur J. Friedel pour les fructueux conseils qu'il nous a donnés.

L'un de nous (P. M.) remercie aussi le Docteur D. A. Degras et M. G. Armand pour les discussions utiles qu'il a eues avec eux.

\section{Bibliographie}

[1] Kittel (C.), Thermal Physics, John Wiley and Sons, Inc., 1969.

[2] Bolt (R. H.), J. Acous. Soc. Am., 1939, 10, 228.

[3] MaA (D. Y.), J. Acous. Soc. Am., 1939, 10, 235.

[4] Montroll (E. W.), J. Chem. Phys., 1950, 18, 183.

[5] Maradudin (A. A.) and Wallis (R. F.), Phys. Rev., 1966, 148, 945.

[6] Dobrzynski (L.) et Leman (G.), J. Physique, 1969, $30,116$.

[7] Patterson (D.), Morrison (J. A.) and ThOMPSON (F. W.), Can. J. Chem., 1955, 33, 240.

[8] Giauque (W. F.) and Archibald (R. C.), J. Am. Chem. Soc., 1937, 59, 561,

[9] Dobrzynski (L.) and Friedel (J.), Surf. Sc., 1968, $12,469$.

[10] FrIEDEL (J.), dans : The interaction of Radiation with Solids, Summer School (Moll, Belgium, 1963), Eds. R. Strumane et al. (North-Holland Publ. Co. Amsterdam, 1964).

[11] FRIEDEL (J.), dans : Summer School on Radiation Damage (Ispra, Italy, 1962), (Gordon and Breach, N. Y.).

[12] Semenchenko (V. K.), dans : Surface Phenomena in Metals and Alloys, Ed. R. Kennedy (Pergamon Press, London).

[13] Funk (E. R.), Udin (H.) and WulfF (J.), J. Metals, $1951,3,1206$

[14] Buttner (F. H.), Udin (H.) and WulfF (J.), $J$. Metals, 1951, 3, 1209.

[15] Udin (H.), Shaler (A. I.) and WulfF (J.), J. Metals, $1949,1,1936$

[16] Burton (W. K.) and Cabrera (N.), Dis. Faraday Soc., 1949, 5, 33

[17] Bassett (G. A.), Phil. Mag., 1958, 3, 1042.

[18] Allpress (J. G.) and SAnders (J. V.), Phil. Mag., $1964,9,645$.

[19] Bethat (H.), Surf. Sc., 1964, 3, 33.

[20] Bahl (O. P.), Evans (E. L.) and Thomas (J. M.), Surf. Sc., 1967, 8, 473.
[21] Cabrera (N.) and Burton (W. K.), Disc. Faraday Soc., 1959, 28, 23.

[22] Bassett (G. A.), Nature, Lond., 1963, 198, 468.

[23] Ehrlich (G.), dans : Structure and Properties of thin films (New York : John Wiley and Sons, 1959).

[24] Gomer (R.), Disc. Faraday Soc., 1959, 28, 23.

[25] Germer (L. H.) and Mac Rae (A. U.), J. Chem. Phys., 1962, 36, 1555.

[26] Rootsaert (W. J. M.), Von Reijen (L. L.) and Sachtler (W. M. H.), J. Catalysis, 1962, 1, 416.

[27] Allan (G.), (à paraître).

[28] Lifshitz (I. M.) et Rosenzweig (L. N.), Izvest. Akad. Nauk. S. S. S. R., Série Fiz., 1948, 12, 667.

[29] De Witt (B. S.), Phys. Rev., 1956, 103, section III, 1565.

[30] Toulouse (G.), Solid State Comm., 1966, 4, 593.

[31] Friedel (J.), Gautier (G.), Gomes (A. A.) and LenGLART (P.), dans : Quant. Theor. Atoms, 445 (1966).

[32] MASRI (P.) and Dobrzynski (L.) J. Physique, 1971, 32, 939.

[33] Theeten (J. B.) and Dobrzynski (L.) (soumis pour publication à (( Phys. Rev. )).

[34] Montroll (E. W.) and Potts (R. B.), Phys. Rev., $1955,100,525$.

[35] Lifschitz (M.), Nuovo Cimento, 1956, suppl. al volume III, série $X, \mathbf{4}, 716$.

[36] Ludwig (W.) and Lengeler (B.), Solid State Comm. 1964, $2,83$.

[37] Dobrzynski (L.) and Mills (D. L.), J. Phys. Chem. Solids, 1969, 30, 1043.

[38] Mills (D. L.), J. Physique, 1967, suppl. au no 2, 28, C-1.

[39] Salter (L.), Proc. Roy. Soc., 1956, 233, 418.

[40] Cyrot-Lackmann (F.), J. Phys. Chem. Solids, 1968, 29,1235 . 